\title{
Unperturbed Chain Dimension of Branched Polypeptides. II. Comb-Like Polypeptides
}

\author{
Masahito OKA and Akio NAKAJIMA \\ Department of Polymer Chemistry, Kyoto University, Sakyo-ku, \\ Kyoto 606, Japan.
}

(Received May 7, 1977)

\begin{abstract}
With regard to comb-like polypeptides, theoretical calculations on the mean square of the unperturbed radius of gyration $\left\langle S^{2}\right\rangle_{0, \mathrm{av}}$ and branching factor $g$ in random coil form were carried out for two cases, i.e., uniformly and randomly distributed subchain lengths. The radius of gyration ratio $\left\langle S^{2}\right\rangle_{0, a v} / n l^{2}$ and $g$ were larger than those of the star-like chain of the same number of subchains, and were dependent on the degree of polymerization as well as the number of branching points.
\end{abstract}

KEY WORD Comb-Like Chain / Branched Polypeptide / Unperturbed Dimension / Radius of Gyration / Branching Factor / Uniform Distribution / Random Distribution /

Comb-like polypeptides carrying branches like the feeth of a comb have been regarded as a model of proteins. They were synthesized by three methods. One is the coupling method, ${ }^{1}$ i.e., by coupling the polypeptides to the side chains of a polypeptide backbone. Another is a method using proteins as multifunctional initiators and polymerizing $N$-carboxy- $\alpha$-amino acid anhydrides (NCA) as branching side chains. This method was first performed by Stahmenn and Becker $^{2}$ with crystalline bovine plasma albumin and crystalline chymotrypsin as initiators, and $\mathrm{N}$-carboxyglycine as NCA. After that, there were a number of results ${ }^{3-13}$ showing comb-like chains synthesized by various kinds of proteins and NCAs, as reported by many authors. In such reports, the biological and chemical properties, e.g., antigen-antibody reaction, were investigated. Finally, there is the method $^{15-26}$ of using polylysine, polyornithine, and copolypeptides containing lysine or ornithine as initiators. In this method, comb-like chains were prepared in aqueous solution, ${ }^{15,16,19-26}$ e.g., water-dioxane mixture, and in non-aqueous solution, ${ }^{17,18,22}$ e.g., dioxane. These purely synthetic comb-like polypeptides were used as molecular models in immunology, ${ }^{19,21}$ and of globular proteins. ${ }^{2-26}$ Preliminary work has indicated that comb-like polypeptides are highly compacted conformations.

We calculated the unperturbed dimensions of the comb-like polypeptides in random coil conformation on the basis of the rotational isomeric model $^{27,28}$ as used in the previous $\operatorname{paper}^{29}$ for star-like polypeptides. The unperturbed dimension was calculated as a function of the degree of polymerization, the number of branching point, and the branching factor.

\section{MEAN SQUARE OF THE RADIUS OF GYRATION}

In Figure 1, a comb-like chain consisting of $r$ branching points is shown. Let the number of subchains attached to one branching point be $f$, i.e., branching factor, then the comb-like chain in Figure 1 corresponds to the case of $f=3$. If the total number of subchains in a

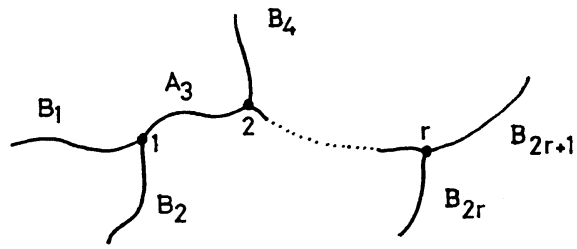

Figure 1. A comb-like chain molecule. 
molecule is $p$, then

$$
p=(f-1) r+1
$$

Subchains are numbered in the order as shown in Figure 1.

We have presented the $i$-th subchain containing branching points on both ends by $A_{i}$, and the $j$-th subchain containing branching point on only one end by $B_{j}$. We designate the numeral $a_{i}$ for subchain $A_{i}$, and $b_{j}$ for subchain $B_{j}$.

$$
\begin{aligned}
& a_{i}=1 \\
& b_{j}=2-f
\end{aligned}
$$

Further, $d_{k h}$ is defined by the following equation.

$$
d_{k h}=(f-2)^{2}\left[\prod_{\left(i_{t}, j_{s}\right)} a_{i_{t}} b_{j_{s}}\right]^{-1}
$$

where $\left(i_{t}, j_{8}\right)$ represents all combinations regarding the number of succesive subchains, and $k$ and $h$, respectively, are the minimum and maximum number of subchains in the whole combinations. For example, for the case of the subchains in Figure 1 consisting of succesive subchains $B_{1}$, $A_{3}, A_{5}$, and $B_{6}, d_{16}=b_{1} a_{3} a_{5} b_{6}$.

Let the vector from the $i$-th atom to the $j$-th atom be $\boldsymbol{r}_{i j}$. Similarly to the star-like chains, the square of the radius of gyration of comblike chains is written as

$$
S^{2}=(n+1)^{-2} \sum_{i \leqq k \leqq h \leqq p}^{2} d_{k h}\left[\sum_{0 \leqq i<j \leqq n_{k h}}^{2} r_{i j}^{2}\right]
$$

from Lagrange's theorem and eq 3 . The degree of polymerization of the chain from the $k$-th subchain to the $h$-th subchain is represented by $n_{k h}$. If $k=h$, then $n_{k h}$ is equal to $n_{k}$. Symbolism of the type $\Sigma^{k}$ indicates the serial $k$ symbols of summation. With regard to the rotation around the virtual bonds of its adjacent bond, polypeptide chains can be treated as an independent rotation chain. Thus, the average for all possible conformations may be carried out independently for each subchain. And so eq 4 takes the form

$$
\left\langle S^{2}\right\rangle_{0}=(n+1)^{-2} \sum_{1 \leqq k \leqq h \leqq p}^{2} d_{k h}\left[\sum_{0 \leqq i<j \leqq n_{k h}}^{2}\left\langle r_{i j}^{2}\right\rangle\right] .
$$

When all the subchains are statistically equal, eq 5 can be simplified as

$$
\begin{aligned}
\left\langle S^{2}\right\rangle_{0}= & {\left[(f-1)^{2}{ }_{0 \leqq i<j \leqq n_{k_{1}}+n_{k 2}+\cdots+n_{k r+1}} \sum_{i j}^{2}\right\rangle } \\
& -\frac{(f-1)(f-2)}{2} r r_{0 \leqq i<j \leqq n_{k}+n_{h}}\left\langle r_{i j}^{2}\right\rangle \\
& \left.-f(f-2) \sum_{0 \leqq i<j \leqq n_{k}}^{2}\left\langle r_{i j}^{2}\right\rangle\right](n+1)^{-2}
\end{aligned}
$$

\section{AVERAGE ON THE DISTRIBUTION OF DEGREES OF POLYMERIZATION IN SUBCHAINS}

From eq 5 or 6 , the unperturbed dimension of the comb-like chains can be calculated if the degree of polymerization is known, by using eq 5 and 6 in Part $\mathrm{I}^{29}$ of this series or eq 5 and 7 in Part I. (Hereafter, Part I will be used for reference simplicity.) However, it seems almost impossible to know the degrees of polymerization of all the subchains.

The average is carried out for two cases of statistical interest. In one case is the average on the uniform distribution of subchain lengths, and the other is that on the random distribution of subchain lengths.

\section{Uniform Distribution}

Let the degree of polymerization of the branched molecule be $n$, and that of the $k$-th subchain be $n_{k}$. Since the degree of polymerization of all the subchain are assumed to be equal, $n_{k}$ is represented by eq 9 in Part I. In the case of uniform distribution of subchain lengths, using eq 9 in Part I, we obtain eq 7 from 6 .

$$
\begin{aligned}
\left\langle S^{2}\right\rangle_{0, \mathrm{av}}= & (n+1)^{-2}\left[(f-1)^{2} \sum_{0 \leqq i<j \leqq(r+1) n_{k}}\left\langle r_{i j}^{2}\right\rangle\right. \\
& -\frac{(f-1)(f-2)}{2} r_{0 \leqq i<j \leqq 2 n_{k}}\left\langle r_{i j}^{2}\right\rangle \\
& \left.-f(f-2) \sum_{0 \leqq i<j \leqq n_{l k}}^{2}\left\langle r_{i j}^{2}\right\rangle\right]
\end{aligned}
$$

The subscript av of $\left\langle S^{2}\right\rangle_{0, \text { av }}$ denotes the average on the distribution on the degree of polymerization of subchains. In a manner similar to the star-like chains, Flory's method ${ }^{27,28}$ is applied to the comb-like chain to calculate the sum of $\left\langle r_{i j}^{2}\right\rangle$ in eq 7. Finally, using method I (see Part I), $\left\langle S^{2}\right\rangle_{0, \text { av }}$ of the comb-like chain composed of subchains with uniform chain length is given by 


$$
\begin{aligned}
& \left\langle S^{2}\right\rangle_{0, \text { av }}
\end{aligned}
$$

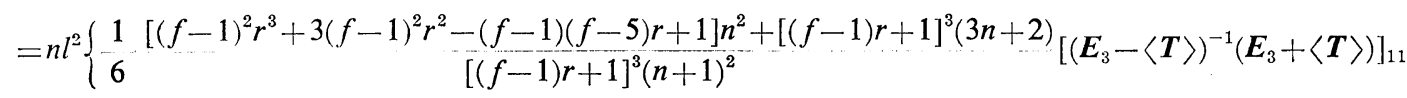

$$
\begin{aligned}
& -(n+1)^{-1}\left[\left(\boldsymbol{E}_{3}-\langle\boldsymbol{T}\rangle\right)^{-2}\langle\boldsymbol{T}\rangle\right]_{11}+2(n+1)^{-2}\left[\left(\boldsymbol{E}_{3}-\langle\boldsymbol{T}\rangle\right)^{-3}\langle\boldsymbol{T}\rangle^{2}\right]_{11} \\
& -2 n^{-1}(n+1)^{-2}\left[( E _ { 3 } - \langle \boldsymbol { T } \rangle ) ^ { - 4 } \langle \boldsymbol { T } \rangle ^ { 3 } \left\{\left(1-\frac{(f-1)(f-2)}{2} r\right) E_{3}-(f-1)^{2}\langle\boldsymbol{T}\rangle^{(r+1) n / p}\right.\right. \\
& \left.\left.\left.+\frac{(f-1)(f-2)}{2} r\langle\boldsymbol{T}\rangle^{2 n / p}+f(f-2)\langle\boldsymbol{T}\rangle^{n / p}\right\}\right]_{11}\right\}
\end{aligned}
$$

Using method II (see Part I), we obtain eq 9. $\left\langle S^{2}\right\rangle_{0, \text { av }}$

$$
\begin{aligned}
\left\langle S^{2}\right\rangle_{0, \mathrm{av}}= & (n+1)^{-2}\left[2(f-1)^{2} \boldsymbol{J}_{7}{ }^{*} D_{1}{ }^{(\langle r+1) n / p)} \boldsymbol{J}_{7}\right. \\
& -(f-1)(f-2) r \boldsymbol{J}_{7}{ }^{*} D_{1}{ }^{(2 n / p)} \boldsymbol{J}_{7} \\
& \left.-2 f(f-2) \boldsymbol{J}_{7}{ }^{*} D_{1}{ }^{(n / p)} \boldsymbol{J}_{7}\right]
\end{aligned}
$$

The symbols in eq 8 and 9 are the same as those in Part I. Equations 8 and 9 include only rotational isomeric approximation, and so are applied to all the comb-like chains whose subchains have the same degree of polymerization. In the case of $r=0$, the comb-like chain is reduced to linear chain. Consequently, in such a case eq 8 and 9 must coinside with the equation for linear chain. Substituting 0 for $r$ in eq 8 and 9 , we obtain the equations for the linear chain by simple calculation. Further, when $r$ is equal to 1 , the comb-like chain becomes a star-like chain whose number of branches is $f$. In the case of $r=1$, eq 8 and 9 , respectively, revert to eq 6 and 7 as in Part I.

\section{Random Distribution}

When the distribution of degree of polymerization of subchains is random, the average of the mean square of radius of gyration is obtained by eq 13 in Part I.

In the case of method I, from eq 6 and eq 5 and 6 in Part $\mathrm{I}$,

$$
\begin{aligned}
\left\langle S^{2}\right\rangle_{0}= & l^{2}(n+1)^{-2}\left\{\frac{1}{6}[n(n+1)(n+2)-r(f-1)(f-2)\right. \\
& \left.\times\left[(f-1) r^{2}+1\right] n_{k} \cdot n_{l} \cdot n_{m}\right] \\
& \times\left[\left(\boldsymbol{E}_{3}-\langle\boldsymbol{T}\rangle\right)^{-1}\left(\boldsymbol{E}_{3}+\langle\boldsymbol{T}\rangle\right)\right]_{11} \\
& -n(n+1)\left[\left(\boldsymbol{E}_{3}-\langle\boldsymbol{T}\rangle\right)^{-2}\langle\boldsymbol{T}\rangle\right]_{11} \\
& +2 n\left[\left(\boldsymbol{E}_{3}-\langle\boldsymbol{T}\rangle\right)^{-3}\langle\boldsymbol{T}\rangle^{2}\right]_{11} \\
& +\left[\left[\{(f-1)(f-2) r-2\} \boldsymbol{E}_{3}\right.\right. \\
& +2(f-1)^{2}\langle T\rangle^{n_{k_{1}}+n_{k_{2}}+\cdots+n_{k}}{ }_{r+1} \\
& \left.-(f-1)(f-2) r\langle\boldsymbol{T}\rangle^{n_{k}+n_{h}}-2 f(f-2)\langle\boldsymbol{T}\rangle^{n_{k}}\right] \\
& \left.\left.\times\left[\left(\boldsymbol{E}_{3}-\langle\boldsymbol{T}\rangle\right)^{-4}\langle\boldsymbol{T}\rangle^{3}\right]\right]_{11}\right\}
\end{aligned}
$$

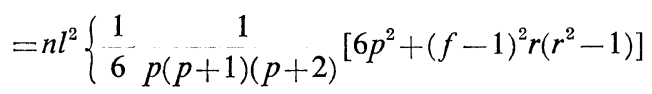

$$
\begin{aligned}
& \times \frac{n+2}{n+1}\left[\left(\boldsymbol{E}_{3}-\langle\boldsymbol{T}\rangle\right)^{-1}\left(\boldsymbol{E}_{3}+\langle\boldsymbol{T}\rangle\right)\right]_{11} \\
& -{ }_{n+1}^{1}\left[\left(\boldsymbol{E}_{3}-\langle\boldsymbol{T}\rangle\right)^{-2}\langle\boldsymbol{T}\rangle\right]_{11} \\
& +\frac{2}{(n+1)^{2}}\left[\left(\boldsymbol{E}_{3}-\langle\boldsymbol{T}\rangle\right)^{-3}\langle\boldsymbol{T}\rangle^{2}\right]_{11} \\
& -\frac{2}{n(n+1)^{2}}\left[1-\frac{(f-1)(f-2)}{2} r\right] \\
& \times\left[\left(\boldsymbol{E}_{3}-\langle\boldsymbol{T}\rangle\right)^{-4}\langle\boldsymbol{T}\rangle^{3}\right]_{11} \\
& +\sum_{i=1}^{(f-1) \boldsymbol{r}} \frac{\mu_{i}\left[\left(\boldsymbol{E}_{3}-\langle\boldsymbol{T}\rangle\right)^{-i-4}\langle\boldsymbol{T}\rangle^{i+3}\right]_{11}}{\prod_{k=-1}^{i}(n-k)(n+1)} \\
& \left.+\sum_{i=(f-2) r}^{(f-1) r} \frac{\nu_{i}\left[\left(\boldsymbol{E}_{3}-\langle\boldsymbol{T}\rangle\right)^{-\imath-4}\langle\boldsymbol{T}\rangle^{n+3}\right]_{11}}{\prod_{k=-1}^{i}(n-k)(n+1)}\right\}
\end{aligned}
$$

where

$$
\begin{aligned}
\mu_{i}= & \frac{2[(f-1) r] !}{[(f-1) r-i] !}(-1)^{i} \\
& \times\left[(f-1)^{2}(-1)^{r+1} \Gamma \frac{\Gamma(i)}{\Gamma(i-r) \Gamma(r+1)}\right. \\
& \left.\quad-\frac{(f-1)(f-2)}{2} r(i-1)+f(f-2)\right] \\
\nu_{i}= & \frac{2[(f-1) r] !}{[(f-1) r-i] !}(-1)^{(f-1) r} \\
\times & {\left[(f-1)^{2}(-1)^{r} \frac{\Gamma(i)}{\Gamma(i-(f-2) r+1) \Gamma((f-2) r)}\right.} \\
+ & (f-1)(f-2) \frac{\Gamma(i)}{2} r_{\Gamma(i-(f-1) r+2) \Gamma((f-1) r-1)} \\
- & \left.f(f-2) \frac{1}{\Gamma(i-(f-1) r+1)}\right]
\end{aligned}
$$

Using eq 10 and 13 in Part $\mathrm{I}$, we obtain eq 11.

To calculate the random average of eq 10 and eq $17-19$ in Part I and following relations are used. 


$$
\begin{aligned}
& \sum_{0<N_{1}<N_{2}<\cdots<N_{p-1}<n}^{p-1} \boldsymbol{A}^{n_{k_{1}+n_{k_{2}}+\cdots+n_{k_{q}}}} \\
& =(-1)^{p+q} \sum_{j=0}^{q-1} p_{p-q+j-1} C_{j} \cdot{ }_{n-p+q-j-1} C_{q-j-1} \\
& \times\left(\boldsymbol{E}_{m}-\boldsymbol{A}\right)^{-p+q-j} \boldsymbol{A}^{n} \\
& +\frac{1}{(p-q-1) !}\left(\boldsymbol{E}_{m}-\boldsymbol{A}\right)^{1-p} \prod_{k=1}^{p-1}(n-k)
\end{aligned}
$$

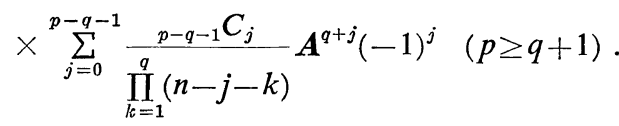

In the case of method II (see Part I), we define $\left\langle C_{r, f}\right\rangle$ by eq 13 .

$$
\left\langle\boldsymbol{C}_{r, f}\right\rangle=\boldsymbol{J}_{7}{ }^{*} \boldsymbol{D}_{1}{ }^{\left({ }_{k_{1}}+n_{k_{2}}+\cdots+n_{k_{r}+1}{ }^{\prime}\right.} \boldsymbol{J}_{\mathbf{7}}
$$

Symbolism of the type $D_{1}^{(n)}$ indicates the serial product of the type $D_{1} D_{2} \cdots D_{n}$, i.e., a product of $n$ such terms. (See ref 27.) Using eq 20 in Part $I$ and eq 13, eq 6 is represented as follows.

$$
\begin{aligned}
\left\langle S^{2}\right\rangle_{0}= & (n+1)^{-2}\left[2(f-1)^{2}\left\langle C_{r, f}\right\rangle\right. \\
& \left.-(f-1)(f-2) r\left\langle B_{p, k h}\right\rangle-2 f(f-2)\left\langle A_{p, k}\right\rangle\right]
\end{aligned}
$$

Substitution of eq 14 into 13 in Part I gives

$$
\begin{aligned}
\left\langle S^{2}\right\rangle_{0, \mathrm{av}}= & (n+1)^{-2}\left[2(f-1)^{2}\left\langle C_{r, f}\right\rangle_{\mathrm{av}}\right. \\
& \left.-(f-1)(f-2) r\left\langle B_{p}\right\rangle_{\mathrm{av}}-2 f(f-2)\left\langle A_{p}\right\rangle_{\mathrm{av}}\right]
\end{aligned}
$$

where $\left\langle C_{r, f}\right\rangle_{\text {av }}$ is defined as random average of $\left\langle C_{r, f}\right\rangle .\left\langle B_{p}\right\rangle_{\mathrm{av}}$ and $\left\langle A_{p}\right\rangle_{\mathrm{av}}$ are obtained in Part I. Calculation of $\left\langle C_{r, f}\right\rangle_{\mathrm{av}}$ is carried out as follows. We define $c_{r, f}$ by following equation.

$$
\begin{aligned}
& \boldsymbol{c}_{r, f}=\sum_{0<N_{1}<\cdots<N_{p-1}<n} \boldsymbol{J}_{7}{ }^{*-1} \boldsymbol{D}_{1}{ }^{\left(N_{1}\right)} \boldsymbol{D}_{N_{1}+1}^{\left(N_{2}-N_{1}\right)} \ldots \\
& \boldsymbol{D}_{N_{r}+1}^{(N)}{ }^{+N^{-1}} \boldsymbol{J}_{7}
\end{aligned}
$$

and

$$
\boldsymbol{c}_{r, f}^{\prime}=\sum_{0<N_{1}<\cdots<N_{r+2}} \boldsymbol{J}_{\mathbf{7}}{ }^{*+1} \boldsymbol{D}_{1}^{\left(N_{2}-N_{1}\right)} \cdots \boldsymbol{D}_{N_{r}+1}^{\left(N_{r+1}-N_{r}\right)} \boldsymbol{J}_{\mathbf{7}}
$$

The sum in this equation is obtained by using following matrix $\{\boldsymbol{L}(k, 2)\}_{i}$

$$
\{\boldsymbol{L}(k, 2)\}_{i}= \begin{cases}\boldsymbol{D}_{i} & (1 \leq i \leq k-1) \\
\boldsymbol{D}_{k} \boldsymbol{I}_{7 k} & (i=k) \\
{\left[\begin{array}{lc}
\boldsymbol{D} & \boldsymbol{I}_{7 \backslash k-1)} \boldsymbol{L}(k-1,2) \\
\mathbf{0} & \boldsymbol{L}(k-1,2)
\end{array}\right]_{i}} & (i \geq k+1)\end{cases}
$$

where

$$
\{\boldsymbol{L}(1,2)\}_{i}=\left[\begin{array}{cc}
\boldsymbol{D} & \boldsymbol{I}_{0} \boldsymbol{H}(2) \\
\mathbf{0} & \boldsymbol{H}(2)
\end{array}\right]_{i} \quad(i \geq \boldsymbol{r}+\mathbf{1})
$$

$\{\boldsymbol{H}(2)\}_{i}$ is represented by eq 23 in Part I, and $\boldsymbol{I}_{k}$ is $7 \times(k+8)$ matrix represented by eq 32 in Part I. Then,

$$
c_{r, f}^{\prime}=\boldsymbol{J}_{7}^{*}\{\boldsymbol{L}(r, 2)\}_{1}^{(n-|f-2| r)} \boldsymbol{J}_{7 r+8}
$$

Substituting this equation into eq 16 , we obtain

$$
c_{r, f}=\sum_{r+1<N_{r+2}<\cdots<N_{p-1}<n} \sum_{7}^{(f-2) r-1} \boldsymbol{J}_{7}^{*}\{\boldsymbol{L}(r, 2)\}_{1}{ }^{(n-r-1)} \boldsymbol{J}_{7 r+8}
$$

Such a summation can be evaluated by using following matrix $\{\boldsymbol{L}(k, h)\}_{i}$

$$
\{\boldsymbol{L}(k, h)\}_{i}=\left\{\begin{array}{lc}
\boldsymbol{D}_{1} & (1 \leq i \leq k-1) \\
\boldsymbol{D}_{k} \boldsymbol{I}_{7 k+h-2} & (i=k) \\
{\left[\begin{array}{cc}
\boldsymbol{L}(k, h-1) & \boldsymbol{L}(k, h-1) \boldsymbol{J}_{7 k+h+5} \\
\multicolumn{1}{c}{\boldsymbol{0}} & 1
\end{array}\right]_{i}} & (i \geq k+1)
\end{array}\right.
$$

where the matrix $\{\boldsymbol{L}(k, h)\}_{i}$ is defined $h \geq 3$. Then, finally we obtain

$$
c_{r, f}=\boldsymbol{J}_{7}^{*}\{\boldsymbol{L}(r,(f-2) r+1)\}_{1}^{(n-(f-2) r)} \boldsymbol{J}_{(f+5) r+7}
$$

From the definition of $\left\langle C_{r, f}\right\rangle_{\mathrm{av}}$

$$
\left\langle C_{r, f}\right\rangle_{\mathrm{av}}=\frac{\boldsymbol{J}_{7}{ }^{*}\{\boldsymbol{L}(r,(f-2) r+1)\}_{1}^{(n-(f-2) r)} \boldsymbol{J}_{(f+5) r+?}}{\sum_{0<N_{1}<\cdots<N_{(f-1) r}<n}^{(f) r} 1}
$$

Using eq 15 and 21, and eq 26 and 30 in Part I, we obtain finally

$$
\begin{aligned}
& \left\langle S^{2}\right\rangle_{0, \text { av }} \\
& =\left[(n+1)^{2} \prod_{i=1}^{(f-1) r}(n-i)\right]^{-1}[(f-1) r] ! \\
& \times\left[2(f-1)^{2} \boldsymbol{J}_{7}{ }^{*}\{\boldsymbol{L}(r,(f-2) r+1)\}_{1}^{(n-|f-2| r)}\right. \\
& \times \boldsymbol{J}_{(f+5) r+7}-(f-1)(f-2) r \\
& \times \boldsymbol{J}_{7}^{*}\{\boldsymbol{K}((f-1) r)\}_{1}^{(n-\{f-1) r+1)} \boldsymbol{J}_{(f-1) r+13} \\
& -2 f(f-2) \boldsymbol{J}_{(f-1) r+7}^{*}\{\boldsymbol{H}((f-1) r+1)\}_{1}{ }^{(n-(f-1) r)} \\
& \left.\times \boldsymbol{J}_{(f-1) r+7}\right]
\end{aligned}
$$

Equations 11 and 22 include only the rotational isomeric approximations, and thus are applied to all the comb-like polypeptides whose subchain lengths distribute randomly. In the case of $r=1$, the comb-like chain is reduced to a star-like chain the number of whose subchains is $f$. 
Substituting 1 for $r$ in eq 11 and 22, we obtain, respectively, eq 16 and 33 in Part I, which are the equations for star-like chain. However, $\{\boldsymbol{L}(1, k)\}_{i}=\{\boldsymbol{K}(k)\}_{i}(k \geq 2)$. In the case of $r=0$ the comb-like chain becomes a linear chain. Defining $\{\boldsymbol{L}(\mathbf{0}, 1)\}_{i}=\boldsymbol{D}_{\boldsymbol{i}}$ and using definition $\{\boldsymbol{H}(1)\}_{i}=\boldsymbol{D}_{i}$ as in Part $\mathrm{I}^{29}$ eq 22 corresponds to eq 7 in Part I. And eq 11 corresponds to eq 6 in Part $\mathrm{I}$.

The branching factor $g$ is defined as the ratio of $\left\langle S^{2}\right\rangle_{0, \text { av }}$ of comb-like chain to $\left\langle S^{2}\right\rangle_{0}$ of the linear chain whose number of bonds are identical with those of the star-like chain. In the case of comb-like chain having a uniformly distributed subchain length, the branching factor $g$ is obtained by dividing eq 8 by eq 6 in Part $I$ or eq 9 by eq 7 in Part $I$. Then, for the infinite degree of polymerization,

$$
g_{\infty}=\frac{(f-1)^{2} r^{3}+3(f-1)^{2} r^{2}-(f-1)(f-5) r+1}{f^{3}}
$$

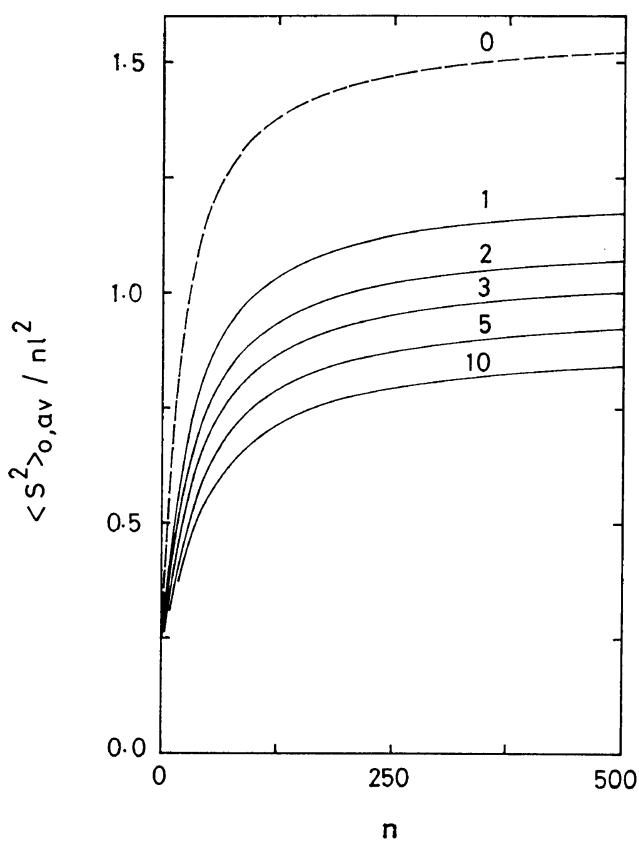

Figure 2. The radius of the gyration ratio $\left\langle S^{2}\right\rangle_{0, \text { av }} / n l^{2}$ plotted against the degree of polymerization $n$ for comb-like polypeptides of $f=3$ having uniform distribution of subchain lengths. The numeral on each curve indicates the number of branching points.
This equation is in accord with the results obtained by Kurata and Fukatsu ${ }^{30}$ for the Gaussian chain. Similarly, in the case of the comb-like chain having randomly distributed subchain lengths, the branching factor $g$ is obtained by dividing eq 11 by eq 6 in Part $\mathrm{I}$, or dividing eq 22 by eq 7 in Part I. Then, for the infinite degree of polymerization,

$$
g_{\infty}=\frac{6 p^{2}+(f-1)^{2} r\left(r^{2}-1\right)}{p(p+1)(p+2)}
$$

This equation is also in accord with the result obtained by Kurata and Fukatsu. ${ }^{30}$

\section{NUMERICAL RESULTS AND DISCUSSION}

Calculations are carried out for comb-like polypeptides composed of $\mathrm{L}$-alanine residues. The value of the transformation matrix $\langle\boldsymbol{T}\rangle$ is the same as that used in our previous paper. ${ }^{29}$

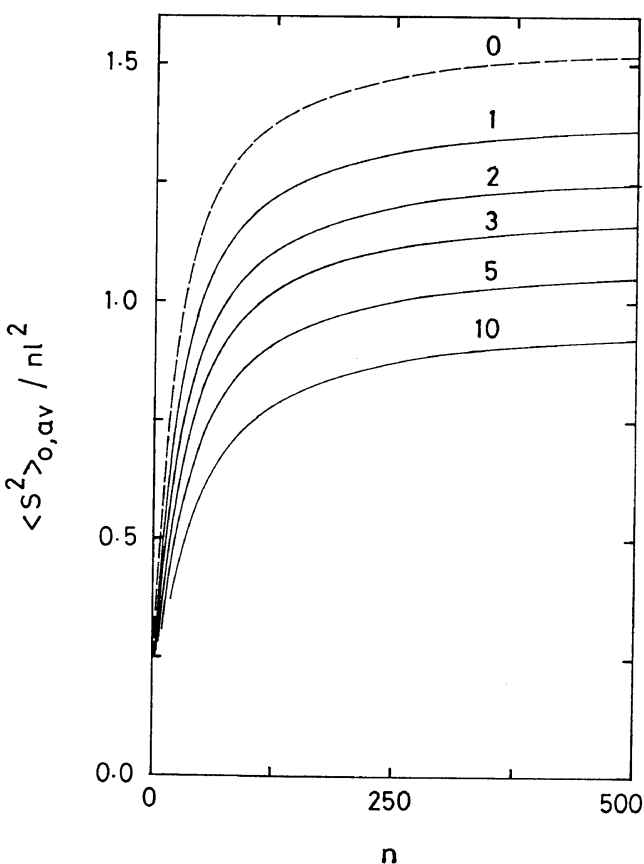

Figure 3. The radius of the gyration ratio $\left\langle S^{2}\right\rangle_{0, a \nabla} / n l^{2}$ plotted against the degree of polymerization $n$ for comb-like polypeptides of $f=3$ having random distribution of subchain lengths. The numeral on each curve indicates the number of branching points. 


\section{The Radius of the Gyration Ratio}

Figure 2 (uniform distribution) and Figure 3 (random distribution) indicate that the radius of the gyration ratio $\left\langle S^{2}\right\rangle_{0, \text { av }} / n l^{2}$ of comb-like polypeptides whose branch lengths distribute uniformly and randomly and whose branching factor $f$ is equal to 3 , increases asymptotically with the degree of polymerization $n$ like that of a linear chain. This tendency is remarkable for the case of random distribution of subchain lengths. Figures 2 and 3 also show that $\left\langle S^{2}\right\rangle_{0, \text { av }} / n l^{2}$ of 3 functional comb-like chain is always smaller than that of linear chain of identical $n$. Comparing Figure 2 with Figure 3, $\left\langle S^{2}\right\rangle_{0, \text { av }} / n l^{2}$ of comb-like chain whose branch lengths distribute uniformly is larger than that of comb-like chain, whose branch lengths distribute randomly and whose number of branching point $r$ is identical to that of uniformly distributed comb-like chain. In the case where $f=4$, Figure 4 (uniform distribution) and Figure

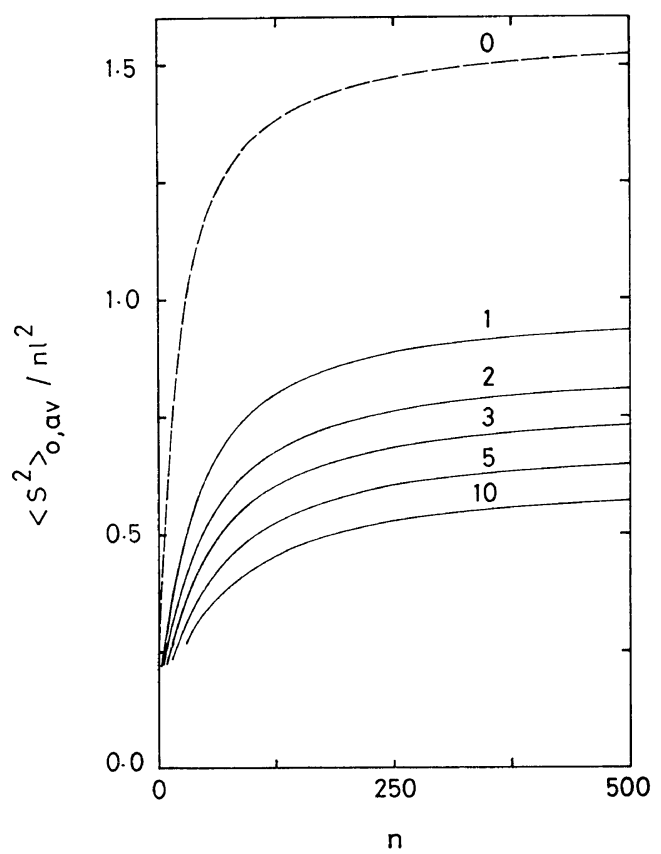

Figure 4. The radius of the gyration ratio $\left\langle S^{2}\right\rangle_{0, a v} / n l^{2}$ plotted against the degree of polymerization $n$ for comb-like polypeptides of $f=4$ having uniform distribution of subchain lengths. The numeral on each curve indicates the number of branching points.

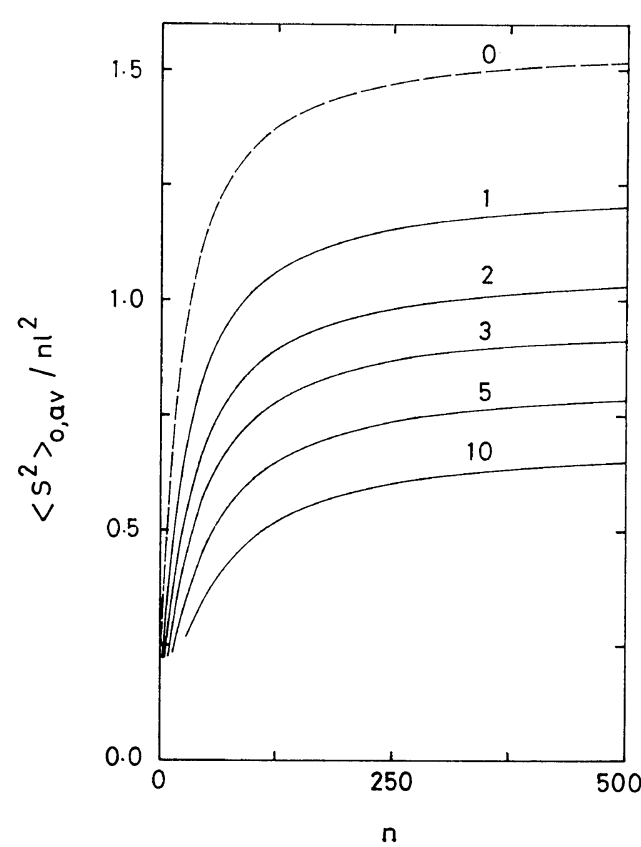

Figure 5. The radius of the gyration ratio $\left\langle S^{2}\right\rangle_{0, \text { a }} / n l^{2}$ plotted against the degree of polymerization $n$ for comb-like polypeptides of $f=4$ having random distribution of subchain lengths. The numeral on each curve indicates the number of branching points.

5 (random distribution) indicate that the same tendency is shown for the case of $f=3$, Figures 6 and 7 indicate that $\left\langle S^{2}\right\rangle_{0, a v} / n l^{2}$ decreases asymptotically with the number of branching point $r .\left\langle S^{2}\right\rangle_{0, a \mathrm{~V}} / n l^{2}$ of comb-like chains is larger than that of star-like chains of identical $p$. The convergence of $\left\langle S^{2}\right\rangle_{0, \text { av }} / n l^{2}$ to the asymptotic value $\left\langle S^{2}\right\rangle_{0, a v} / n l^{2}$ becomes slow with increasing $r$ for $f=3$ and $f=4$ (see Table I). Also $\left\langle S^{2}\right\rangle_{0, \text { av }} / n l^{2}$ of uniform distribution converges more slowly than that of random distribution, but the difference between the value of $r_{s}$ for uniform and random distributions becomes small with increasing $r$.

\section{Branching Factor}

Zimm and Stockmayer, ${ }^{31}$ and Kurata and Fukatsu $^{30}$ showed that the branching factor $g$ of comb-like chain whose branch lengths distribute uniformly and randomly is dependent on the number of branches $p$. In this work Figures $8-11$ show that the branching factor of comb-like chain depends not only on the 
Unperturbed Chain Dimension of Branched Polypeptides. II.

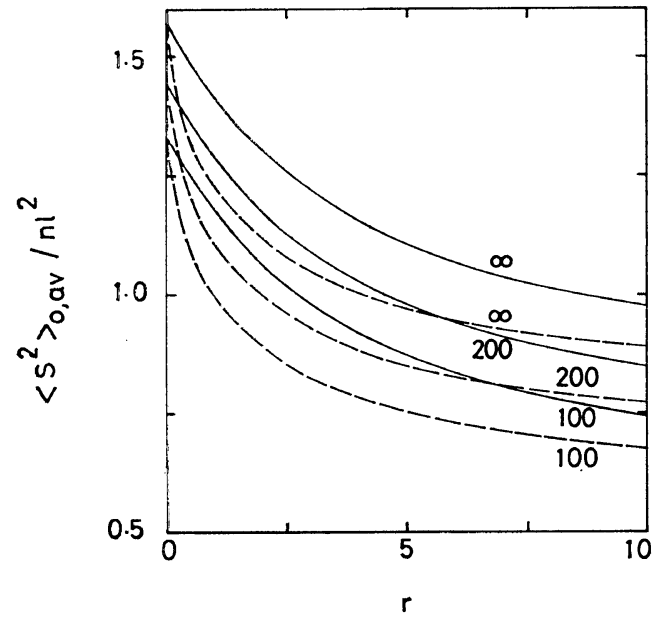

Figure 6. The radius of the gyration ratio $\left\langle S^{2}\right\rangle_{0, \mathrm{av}} / n l^{2}$ plotted against the number of branching points $r$ for comb-like polypeptides of $f=3$ having random distribution of subchain lengths (solid lines), and having uniform distribution of subchain lengths (broken lines). The numeral on each curve indicates the degree of polymerization.

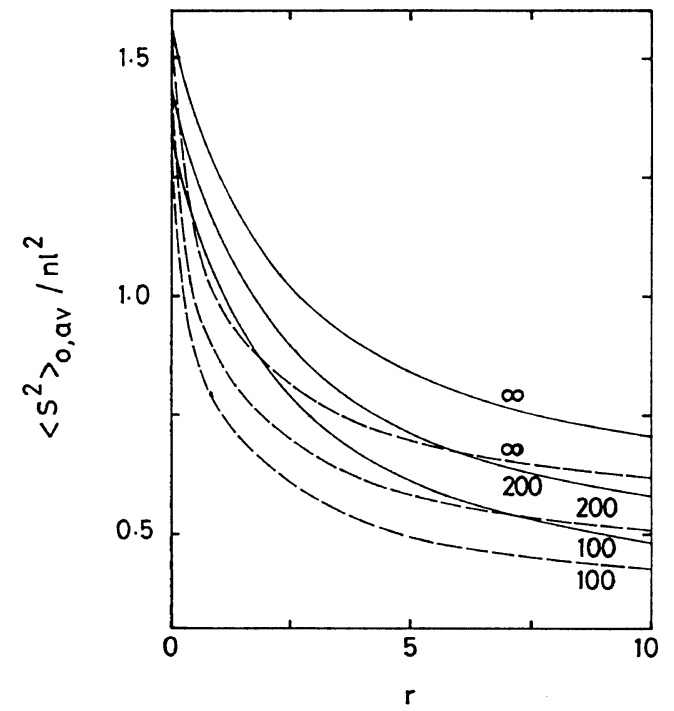

Figure 7. The radius of the gyration ratio $\left\langle S^{2}\right\rangle_{0, a v} / n l^{2}$ plotted against the number of branching points $r$ for comb-like polypeptides of $f=4$ having random distribution of subchain lengths (solid lines), and having uniform distribution of subchain lengths (broken lines). The numeral on each curve indicates the degree of polymerization.

Table Ia. Summary of the number of bonds required for $\left(\left\langle S^{2}\right\rangle_{0, \text { av }} / n l^{2}\right)_{\infty}-\left\langle S^{2}\right\rangle_{0, \text { av }} / n l^{2}$ to be within 10 and $5 \%$ of $\left(\left\langle S^{2}\right\rangle_{0, a v} / n l^{2}\right)_{\infty}$ for comb-like polypeptides

\begin{tabular}{|c|c|c|c|c|c|c|c|c|}
\hline \multirow{3}{*}{$r$} & \multicolumn{4}{|c|}{$f=3$} & \multicolumn{4}{|c|}{$f=4$} \\
\hline & \multicolumn{2}{|c|}{ Uniform } & \multicolumn{2}{|c|}{ Random } & \multicolumn{2}{|c|}{ Uniform } & \multicolumn{2}{|c|}{ Random } \\
\hline & $n(10 \%)$ & $n(5 \%)$ & $n(10 \%)$ & $n(5 \%)$ & $n(10 \%)$ & $n(5 \%)$ & $n(10 \%)$ & $n(5 \%)$ \\
\hline 0 & 157 & 330 & 157 & 330 & 157 & 330 & 157 & 330 \\
\hline 1 & 201 & 414 & 177 & 370 & 248 & 508 & 205 & 420 \\
\hline 2 & 220 & 450 & 194 & 410 & 280 & 581 & 240 & 490 \\
\hline 3 & 231 & 476 & 210 & 440 & 310 & 630 & 270 & 550 \\
\hline 5 & 253 & 517 & 235 & 480 & 352 & 704 & 315 & 640 \\
\hline 10 & 273 & 567 & 265 & 550 & 403 & 806 & 375 & 780 \\
\hline
\end{tabular}

Table Ib. Summary of the number of bonds required for $g_{\infty}-g$ to be within 10 and $5 \%$ of $g_{\infty}$ for comb-like polypeptides

\begin{tabular}{|c|c|c|c|c|c|c|c|c|}
\hline \multirow{3}{*}{$r$} & \multicolumn{4}{|c|}{$f=3$} & \multicolumn{4}{|c|}{$f=4$} \\
\hline & \multicolumn{2}{|c|}{ Uniform } & \multicolumn{2}{|c|}{ Random } & \multicolumn{2}{|c|}{ Uniform } & \multicolumn{2}{|c|}{ Random } \\
\hline & $n(10 \%)$ & $n(5 \%)$ & $n(10 \%)$ & $n(5 \%)$ & $n(10 \%)$ & $n(5 \%)$ & $n(10 \%)$ & $n(5 \%)$ \\
\hline 1 & 33 & 84 & 8 & 34 & 84 & 180 & 37 & 87 \\
\hline 2 & 55 & 120 & 29 & 72 & 119 & 252 & 75 & 160 \\
\hline 3 & 70 & 147 & 46 & 104 & 150 & 310 & 106 & 225 \\
\hline 5 & 88 & 187 & 70 & 151 & 192 & 384 & 152 & 315 \\
\hline 10 & 105 & 231 & 104 & 220 & 248 & 465 & 215 & 450 \\
\hline
\end{tabular}


M. OKA and A. NAKAJIMA

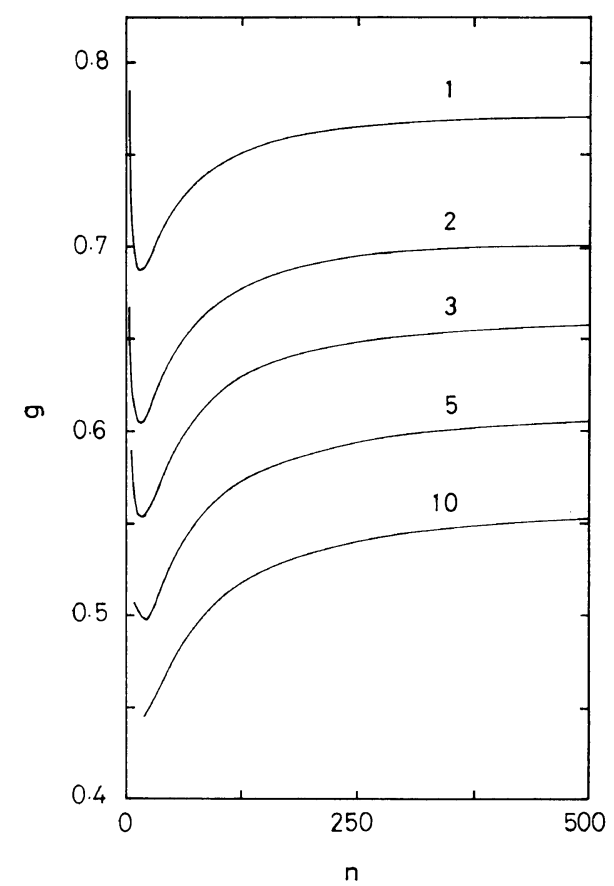

Figure 8. The branching factor $g$ plotted against the degree of polymerization $n$ for comb-like polypeptides of $f=3$ having uniform distribution of subchain lengths. The numeral on each curve indicates the number of branching points.

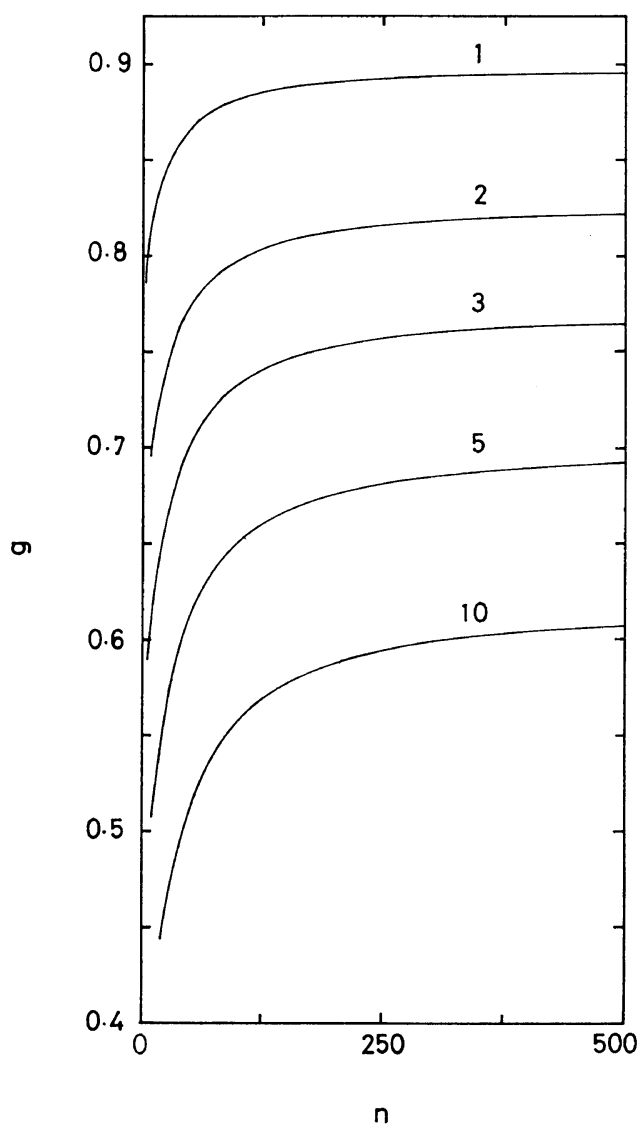

Figure 9. The branching factor $g$ plotted against the degree of polymerization $n$ for comb-like polypeptides of $f=3$ having random distribution of subchain length. The numeral on each curve indicates the number of branching points. 
Unperturbed Chain Dimension of Branched Polypeptides. II.

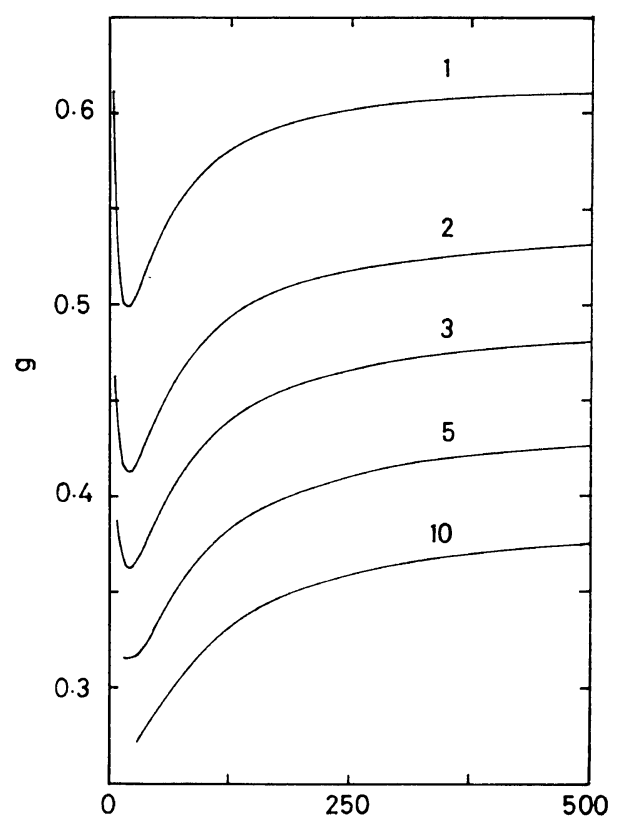

Figure 10. The branching factor $g$ plotted against the degree of polymerization $n$ for comb-line polypeptides of $f=4$ having uniform distribution of subchain lengths. The numeral on each curve indicates the number of branching points.

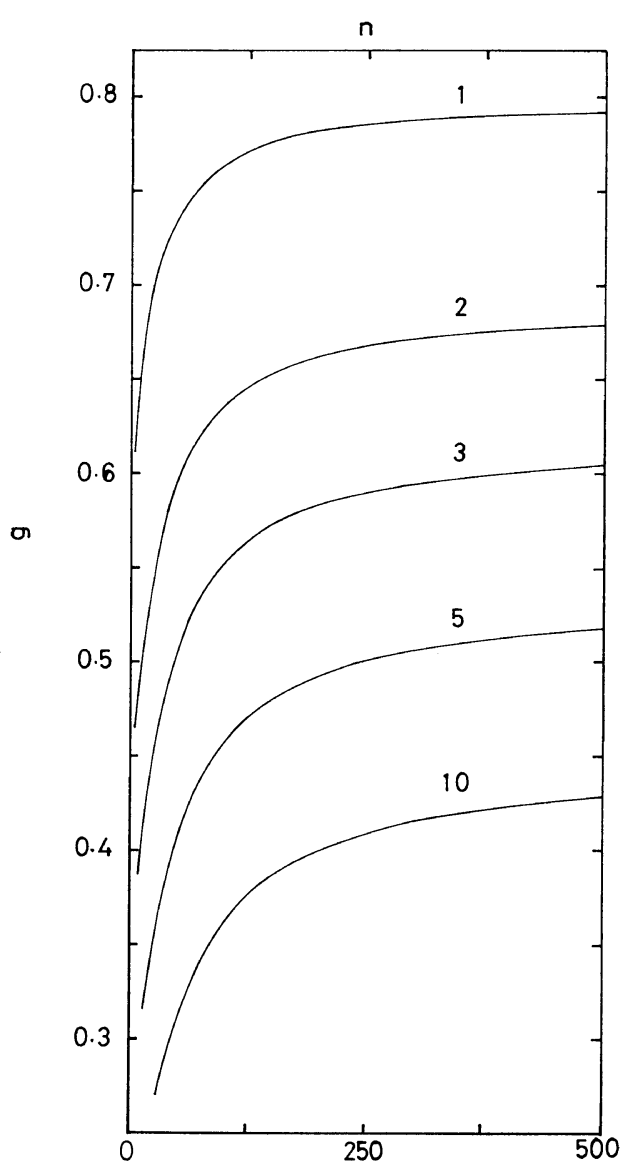

Figure 11. The branching factor $g$ plotted against the degree of polymerization $n$ for comb-like polypeptides of $f=4$ having random distribution of subchain lengths. The numeral on each curve indicates the number of branching points. 


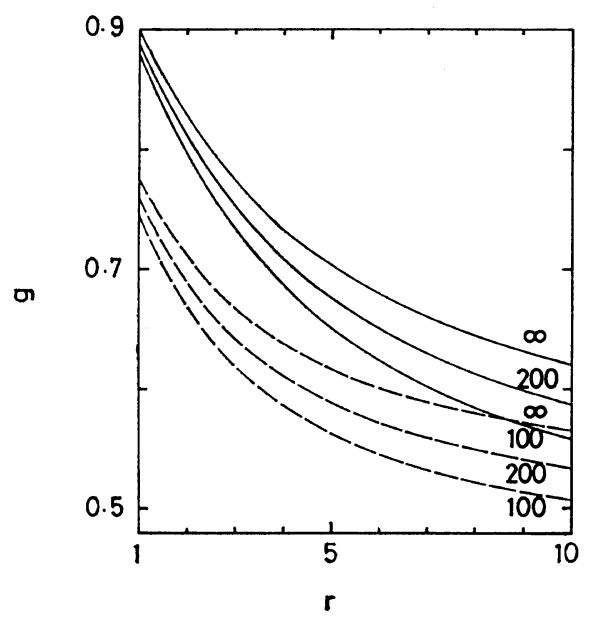

Figure 12. The branching factor $g$ plotted against the number of branching points $r$ for comb-like polypeptides of $f=3$ having random distribution of subchain lengths (solid lines), and having uniform distribution of subchain length (broken lines). The numeral on each curve indicates the degree of polymerization.

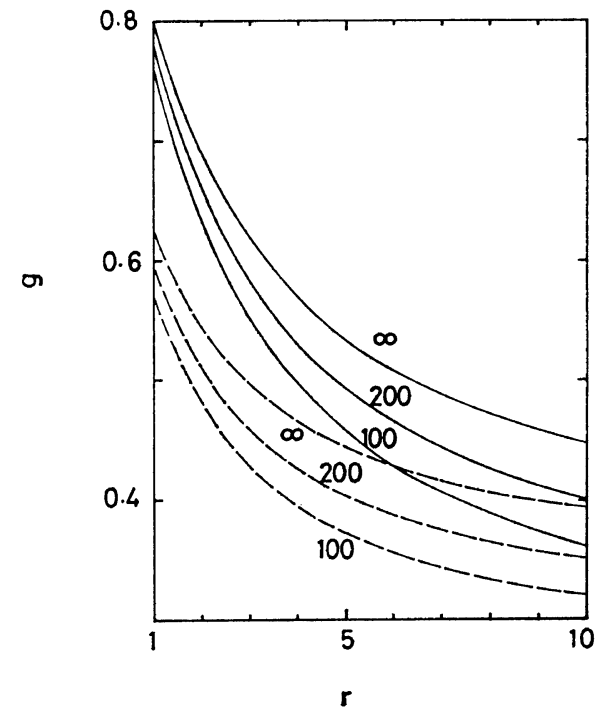

Figure 13. The branching factor $g$ plotted against the number of branching points $r$ for comb-like polypeptides of $f=4$ having random distribution of subchain lengths (solid lines), and having uniform distribution of subchain lengths (broken lines). The numeral on each curve indicates the degree of polymerization.
Table II. Summary of the results on $g_{\mathrm{min}}$ for comb-like polypeptides whose subchain lengths distribute uniformly

\begin{tabular}{ccccc}
\hline & \multicolumn{2}{c}{$f=3$} & \multicolumn{2}{c}{$f=4$} \\
& $n\left(g_{\text {min }}\right)^{\mathrm{a}}$ & $g_{\text {min }}$ & $n\left(g_{\text {min }}\right)$ & $g_{\text {min }}$ \\
\hline 1 & 15 & 0.685 & 16 & 0.499 \\
2 & 15 & 0.605 & 21 & 0.413 \\
3 & 21 & 0.554 & 20 & 0.362 \\
4 & 18 & 0.519 & 26 & 0.333 \\
5 & 22 & 0.498 & - & - \\
6 & 26 & 0.485 & - & - \\
\hline
\end{tabular}

a The number of bonds at which $g$ obtains the minimum value $g_{\mathrm{min}}$.

number of branches $p$, but also on the degree of polymerization $n$. In the case of random distribution, the value of $g$ increases monotonously to the asymptotic limit, i.e., $g_{\infty}$. In the case of uniform distribution, $g$ increases monotonously to $g_{\infty}$ when $r>6$ for $f=3$ and $r>4$ for $f=4$, but $g$ decreases with $n$, and passes through a minimum, i.e., $g_{\mathrm{min}}$, and increases toward $g_{\infty}$ with $n$ when $r \leq 6$ for $f=3$ and $r \leq 4$ for $f=4$. The value of $g_{\mathrm{min}}$ decreases with $r$ for $f=3$ and $f=4 . g$ converges more radidly to $g_{\infty}$ with increasing $n$ than $\left\langle S^{2}\right\rangle_{0, \text { av }} / n l^{2}$ in both cases of uniform and random distributions. In the case of random distribution $g$ converges more rapidly to $g_{\infty}$ with increasing $n$ than $g$ for uniform distribution, but the difference in the value $g / g_{\infty}$ between uniform and random distributions becomes small with increasing $r$, as with $\left\langle S^{2}\right\rangle_{0, \text { av }} / n l^{2}$.

Tonelli $^{32}$ has calculated the value of $g$ for particular star-like and comb-like polyethylenes whose subchain length $n_{k}$ is specified. As is shown in Table III, Tonelli's results are slightly different from our results, but the difference between the two values of $g$ is within $3 \%$.

The hydrodynamic properties ${ }^{22,24}$ and the light scattering results ${ }^{23}$ in helicogenic solvent (DMF) showed that the comb-like polypeptides form a very compact conformation in solution. In this work we have also shown that comb-like polypeptides in random coil conformation have a very compact form compared with linear polypeptides having the same degree of polymerization.

Computations were carried out by the FACOM 230-75 computer at the Kyoto University Computation Center. 
Unperturbed Chain Dimension of Branched Polypeptides. II.

Table III. Summary of the results on $g$ for comb-like polypeptides whose subchain lengths distribute uniformly

\begin{tabular}{|c|c|c|c|c|}
\hline \multirow{2}{*}{$r$} & \multicolumn{4}{|c|}{$f=3$} \\
\hline & $n_{k}=25$ & $n_{k}=50$ & $n_{k}=100$ & $n_{k}=\infty$ \\
\hline 1 & $0.735(0.737)^{a}$ & $0.756(0.769)$ & $0.767(0.769)$ & 0.778 \\
\hline 2 & $0.678(0.684)$ & $0.695(0.699)$ & $0.703(0.690)$ & 0.712 \\
\hline 3 & $0.639(0.654)$ & $0.653(0.657)$ & $0.661(0.661)$ & 0.668 \\
\hline 4 & $0.614(0.619)$ & $0.626(0.612)$ & $0.632(0.642)$ & 0.638 \\
\hline 5 & $0.596(0.602)$ & $0.606(0.597)$ & $0.612(0.620)$ & 0.617 \\
\hline \multirow{2}{*}{$r$} & \multicolumn{4}{|c|}{$f=4$} \\
\hline & $n_{k}=25$ & $n_{k}=50$ & $n_{k}=100$ & $n_{k}=\infty$ \\
\hline 1 & $0.571(0.568)$ & $0.597(0.615)$ & $0.611(0.608)$ & 0.625 \\
\hline 2 & $0.507(0.520)$ & $0.526(0.530)$ & $0.535(0.530)$ & 0.545 \\
\hline 3 & $0.466(0.479)$ & $0.481(0.474)$ & $0.488(0.502)$ & 0.496 \\
\hline 4 & $0.440(0.445)$ & $0.452(0.447)$ & $0.459(0.469)$ & 0.465 \\
\hline 5 & $0.423(0.416)$ & $0.433(0.430)$ & $0.438(0.446)$ & 0.443 \\
\hline
\end{tabular}

a Numerical values in parentheses are Tonelli's results obtained with $\sigma=0.54, \phi=1$, and $\omega=0.088$ for 3 and 4 functional comb-like polyethylenes.

\section{REFERENCES}

1. M. Bovarnick, F. Eisenberg, Jr., D. O'Connell, J. Victor, and P. Owades, J. Biol. Chem., 207, 593 (1954).

2. M. A. Stahmann and R. R. Becker, J. Am. Chem. Soc., 74, 2695 (1952).

3. H. F. Conrat, Biochim. Biophys. Acta, 10, 180 (1953).

4. A. Stracher, W. H. Konigsberg, and R. R. Becker, ibid., 20, 595 (1956).

5. R. R. Becker and M. A. Stahmann, J. Biol. Chem., 204, 745 (1953).

6. M. Green and M. A. Stahmann, ibid., 213, 259 (1955).

7. H. Tsuyuki, H. V. Kley, and M. A. Stahmann, J. Am. Chem. Soc., 78, 764 (1956).

8. H. V. Kley and M. A. Stahmann, J. Phys. Chem., 60, 1200 (1956).

9. M. Sela and F. Haurowitz, Experientia, 14, 91 (1958).

10. W. H. Konigsberg and R. R. Becker, J. Am. Chem. Soc., 81, 1428 (1959).

11. A. Stracher and R. R. Becker, ibid., 81, 1432 (1959).

12. M. Sela and R. Arnon, Biochem. J., 75, 91 (1960).

13. R. Arnon and M. Sela, ibid., 75, 103 (1960).

14. M. Sela and R. Arnon, ibid., 77, 394 (1960).

15. M. Sela and E. Katchalski, Experientia, 11, 62 (1955).

16. M. Sela, E. Kathalski, and M. Gehatia, J. Am. Chem. Soc., 78, 746 (1956).
17. A. Yaron and A. Berger, Bull. Res. Counc. of Israel, 7A, 96 (1958).

18. A. Yaron and A. Berger, ibid., 9A, 117 (1960).

19. M. Sela and R. Arnon, Biochim. Biophys. Acta, 40, 382 (1960).

20. N. Lupe, A. Yaron, M. Sela, and A. Berger, Bull. Res. Counc. of Israel, 10A, 47 (1961).

21. M. Sela, S. Fuchs, and R. Arnon, Biochem. J., 85, 223 (1962).

22. A. Yaron and A. Berger, Biochim. Biophys. Acta, 107, 307 (1965).

23. E. Eliezer and A. Silberberg, Biopolymers, 5, 95 (1967).

24. N. Eliezer and A. Silberberg, ibid., 5, 105 (1967).

25. J. P. Williot, J. M. Ruysschaert, and J. Jaffe, J. Polym. Sci. (Phys.), 10, 2125 (1972).

26. A. Berger and A. Yaron, "Polyamino Acids, Polypeptides, and Proteins," Univ. of Wisconsin Press, Madison, Wisconsin, 1962, p 13.

27. P. J. Flory, "Statistical Mechanics of Chain Molecules," John Wiley \& Sons, Inc., New York, N.Y., 1969.

28. P. J. Flory, Macromolecules, 7, 381 (1974).

29. M. Oka and A. Nakajima, Polym. J., 9, 573 (1977).

30. M. Kurata and M. Fukatsu, J. Chem. Phys., 41, 2934 (1964).

31. B. H. Zimm and W. H. Stockmayer, ibid., 17, 1301 (1949).

32. A. E. Tonelli, J. Am. Chem. Soc., 94, 2972 (1972). 